\title{
La normalisation en management de projet : comprendre les corpus dominants de connaissances dans la discipline
}

\author{
Serge Nenkam, Doctorant en management de projets \\ Caroline Gagné, Ph. D. \\ Université du Québec à Chicoutimi
}

\section{INTRODUCTION}

Les fondements du management de projet ne sont pas nouveaux. Dans sa forme moderne, le management de projet fait l'objet d'une multitude de corpus de connaissances.

Les fondements du management de projet ne sont pas nouveaux. Dans sa forme moderne, le management de projet fait l'objet d'une multitude de corpus de connaissances. De fait, une revue de littérature des articles publiés entre 1990 et 2013 par le Project Management Journal, l'International Journal of Project Management, La Cible, le Project Management Network, révèle que les chercheurs en milieu universitaire et les professionnels en management de projet se préoccupent de plus en plus de la problématique créée par l'abondance de ces différents courants de pensées et conceptions épistémologiques. Cette profusion idéologique entraine avec elle deux problèmes majeurs. Primo, les professionnels en pratique ont l'ambiguïté de choix quand il s'agit de déterminer le guide, le support ou le corpus de connaissances devant faciliter la réalisation des projets. Secundo, en théorie, chercheurs et universitaires se livrent une bataille sans merci pour positionner, contrôler, voire imposer aux recherches le corpus de leur choix. Pourtant, nous pensons que le choix d'un courant de pensée, d'un corpus de connaissances ou d'une famille professionnelle devrait relever d'un ensemble de considérations importantes : l'expérience professionnelle, la localisation géographique et la reconnaissance internationale.

Cet article a pour but de contribuer à la compréhension des corpus dominants de connaissances dans la discipline du management de projet. Ainsi, après avoir présenté une brève genèse $\mathrm{du}$ management de projet et les critères de son acceptation en tant qu'épistémologie doctrinale, nous tentons d'établir objectivement les articulations des principaux corpus de connaissances qui prolifèrent sur le terrain. Enfin, nous présentons la norme ISO 21500 qui situe les lignes directrices sur le management de projet comme une alternative normative à ces différents corpus de connaissances.

\section{BRÈVE GENÈSE DU MANAGEMENT DE PROJET}

Si l'on considère le management comme l'art de gérer, de diriger, d'organiser et de contrôler, et si l'on regarde les projets de manière simpliste comme étant des initiatives temporelles, on peut soutenir que le management de projet est né depuis que l'humain existe. Pendant longtemps, les êtres humains entreprenaient et exerçaient des activités temporelles pour subvenir à leurs besoins. De la chasse à la pêche en passant par la cueillette, les premiers humains satisfaisaient leurs besoins vitaux en mettant en œuvre des projets pour leur survie. Pendant la période antique, on a assisté à la réalisation des grands ouvrages prodigieux, parfois sous des contraintes concurrentes persistantes. On peut citer, par exemple, la construction de la pyramide de Khéops en Égypte encore appelée la grande pyramide de Gizeh vers l'an 2560 av. J.-C., la construction des jardins suspendus de Babylone en Irak qui date du $\mathrm{VI}^{\mathrm{e}}$ siècle av. J.-C., la gravure de la statue Chryséléphantine de Zeus en Grèce ou encore le mausolée d'Halicarnasse en Turquie à l'an 353 av. J.-C. Comment ne pas affirmer que la réalisation de ces œuvres gigantesques relevait de la maîtrise des outils de gestion sophistiqués et de la capacité à gérer, à diriger, à organiser et à contrôler les ressources disponibles? Il est certain que les pratiques de gestion antiques étaient adaptées à cette ère pour favoriser la réalisation de ces types de projets. Nous pouvons comprendre qu'il aurait parfois été possible de faire dans l'improvisation et 
le tâtonnement. De même, nous pouvons également entrevoir que l'absence de certaines technologies et de certains outils de l'information et des communications contribuait à alourdir les durées, les coûts et l'utilisation des ressources. Une chose est certaine, la mise en œuvre des grands projets antiques relevait du management de projet. De ce point de vue, il ne serait pas erroné de remonter l'histoire du management de projet bien plus tôt, puisqu'opérationnellement, ces travaux nécessitaient de grandes capacités intellectuelles dans les domaines de la planification, de l'exécution et du contrôle des ressources humaines et matérielles.

En ce qui s'agit du management de projet moderne, que nous considérons comme étant plus élaboré et mieux structuré, il est possible de situer ses origines depuis l'époque où Henri Fayol (1916) ${ }^{1}$ a défini les cinq fonctions principales d'un manager : planifier, organiser, diriger, coordonner et contrôler (PODC). Certains auteurs ${ }^{2}$ pensent plutôt que le management de projet moderne est plus récent et puise ses origines de l'avant-Deuxième Guerre mondiale, lorsqu'il fallait développer des grands projets de création de missiles de guerre et mettre en œuvre la bombe atomique. De même ${ }^{3}$, le management de projet moderne est né de la création de la méthode du chemin critique (Critical Path Method/ Program Evaluation Review Technique - CPM/ PERT) dans les années 1950. Quoi qu'il en soit, s'il faut parler du management de projet tel qu'il est perçu aujourd'hui, sous une forme beaucoup plus systémique, il semble pertinent de considérer le découpage historique élaboré ${ }^{4}$. En effet, Kwak considère que l'histoire du management de projet moderne peut être décomposée en quatre (4) temps.

La première période n'est rien d'autre que celle des années 1900 à 1957 . Elle a été stimulée par une série d'innovations technologiques étalées au long du $19^{\mathrm{e}}$ siècle. C'est dans cette période que Henry Gantt a inventé le Diagramme de Gantt ${ }^{5}$. C'est également dans cette période qu'Henri Fayol ${ }^{1}$ a publié les fonctions d'un manager et développé les 14 principes du management. De même, la structuration des activités dans un projet a permis de mettre sur pied le concept de "structure de découpage du projet » (SDP). La théorie de Monte Carlo a également permis d'effectuer des simulations pour l'estimation des risques et des délais dans les projets. La deuxième période va de 1958 à 1979 et peut être considérée comme l'ère du management de projet scientifique. La création des associations professionnelles de management de projet a ouvert la voie à une profusion de modèles et de corpus de connaissances dans la discipline. L'International Project Management Association (IPMA ${ }^{\circledR}$ ) pose ses fondements en 1964, le Project Management Institute $\left(\mathrm{PMI}^{\circledR}\right)$ est créé en 1969. L'invention du CPM/PERT a donné la possibilité d'obtenir de plus en plus des réductions sur les durées de réalisation des projets. La structuration des bureaux de projet a encouragé les entreprises à opter davantage pour des structures organisationnelles matricielles avec une mixité des rôles et responsabilités entre les responsables fonctionnels et les chefs de projet.

La troisième période, celle de 1980 à 1994, est marquée par un grand intéressement des chercheurs qui ont multiplié les thèmes de recherches pour faire avancer la discipline. Ainsi, les chercheurs de l'IPMA $^{\circledR}$, par exemple, prenaient le pas sur le management des parties prenantes lorsque ceux de l'Association of Project Management (APM ${ }^{\circledR}$ ) développaient le management par exception.

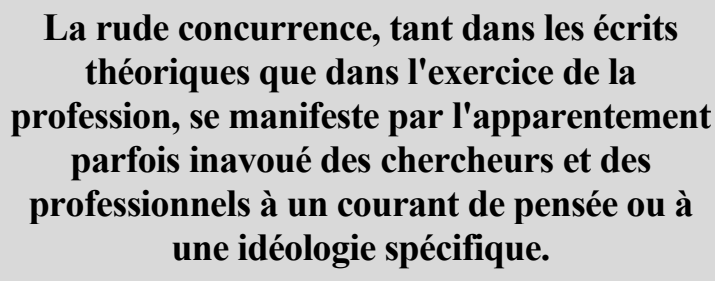

La dernière période, celle de 1995 à nos jours, peut être considérée comme la période pendant laquelle la discipline a atteint sa véritable vitesse de croisière. Elle est marquée par deux évènements majeurs. D'abord l'élaboration de la théorie de la chaîne critique, ensuite la rude concurrence à la fois sur le champ théorique que pratique des différents courants de pensées et des modèles de management de projet. La théorie de la chaîne critique, a permis d'optimiser la démarche CPM/PERT ${ }^{6}$. La rude concurrence, tant dans les écrits théoriques que dans l'exercice de la profession, se manifeste par l'apparentement parfois inavoué des chercheurs et des professionnels à un courant de pensée ou à une idéologie spécifique.

Tel que présenté, nous constatons que le management de projet a connu une évolution remarquable. Cependant, la question de son épistémologie doctrinale a parfois suscité quelques préoccupations. 


\section{LE QUESTIONNEMENT ÉPISTÉMOLOGIQUE DU MANAGEMENT DE PROJET}

De nos jours, la littérature scientifique
soutien désormais unanimement que le
management de projet constitue une
discipline à part entière. Sa démarche repose
sur un processus rigoureux et systématique
qui obéit à une structuration de l'esprit sur le
plan théorique et à l'utilisation des outils et
des techniques pour mettre en pratique cet
esprit.

Plusieurs ambiguïtés ont longtemps demeuré quant au statut du management de projet en tant qu'une épistémologie doctrinale. Le Moigne $(1990)^{7}$ énumère trois principes fondamentaux pour qualifier une discipline : le principe ontologique, le principe de l'univers câblé et le principe de l'objectivité.

Le principe ontologique porte sur la science de l'être, de ce qui est. Le management de projet en tant qu'être se manifeste par son approche systémique, constituée du dualisme intrants/extrants.

Le management de projet obéit également au principe de l'univers câblé. L'objet de recherche préexiste et ne dépend pas du chercheur. Il existe un lien de cause à effet entre les différents phénomènes étudiés.

Enfin, le principe de l'objectivité en management de projet se manifeste par la neutralité du chercheur envers son objet de recherche. Toute interaction ou prise de position subjective peut nuire à la réalité ontologique. Le réel existe indépendamment du phénomène observé.

De nos jours, la littérature scientifique soutien désormais unanimement que le management de projet constitue une discipline à part entière ${ }^{8}$. Sa démarche repose sur un processus rigoureux et systématique qui obéit à une structuration de l'esprit sur le plan théorique et à l'utilisation des outils et des techniques pour mettre en pratique cet esprit. Plusieurs corpus de connaissances se sont développés au fil des années. La majorité de ceuxci relève du positivisme puisque des lignes directrices, des outils et techniques et parfois des compétences sont établis à un niveau général pour être appliqués dans des projets spécifiques. La discipline du management de projet a ses concepts, mais les méthodes de leurs mises en œuvre varient d'un corpus de connaissances à un autre.

\section{LES CORPUS DOMINANTS DE CONNAISSANCES EN MANAGEMENT DE PROJET}

\section{Les corpus de connaissances sont davantage différents sur les modèles de gestion des projets que sur les concepts, mais utilisent les mêmes outils et techniques, puisque ces outils ont été élaborés et testés dans d'autres domaines de gestion tels que l'Économie, le Droit ou la Finance.}

Si en théorie il existe une diversité d'opinions, de visions ou de corpus de connaissances dans la discipline du management de projet, il faut reconnaître que dans la pratique par contre, toutes ces différences conceptuelles se chevauchent. La plupart des chercheurs et des professionnels sont par exemple d'accord sur les éléments qui définissent un projet : initiative temporelle et unique réalisée suivant certaines contraintes (contenu, délais, coûts, risques, qualité, ressources, rendement) ayant pour but de satisfaire les exigences des parties prenantes et d'apporter une plus-value à l'organisation.

Les corpus de connaissances sont davantage différents sur les modèles de gestion des projets que sur les concepts, mais utilisent les mêmes outils et techniques, puisque ces outils ont été élaborés et testés dans d'autres domaines de gestion tels que l'Économie, le Droit ou la Finance. C'est le cas par exemple de la technique du benchmarking encore appelée étalonnage, qui provient de la mercatique et qui est très utilisée pour la gestion de la qualité consistant à analyser les modes de gestion des entreprises du même secteur et de s'en inspirer afin d'en tirer un meilleur avantage.

Trois corpus de connaissances dominent le terrain pratique et méthodologique de l'environnement international du management de projet ${ }^{8}$. 
Pour valider les acquis professionnels et pratiques de ses membres, le PMI ${ }^{\circledR}$ propose sept certifications professionnelles basées sur des aspects technico-pratiques du management de projet.

Le premier corpus est tenu par le Project Management Institute $\left(\mathrm{PMI}^{\circledR}\right)$ à travers son corpus de connaissances en management de projet, le guide $\mathrm{PMBOK}^{\circledR}$. $\mathrm{PMI}^{\circledR}$, dont le siège est situé aux ÉtatsUnis, revendique 438857 membres dans 193 pays au 30 septembre $2014^{9}$. Le management de projet selon PMI ${ }^{\circledR}$ adopte l'approche processus et s'articule autour de 47 processus segmentés en cinq groupes $^{10}$ : initiation, planification, exécution, surveillance et maîtrise, clôture. Ces groupes de processus transcendent dix domaines de connaissance : l'intégration, le contenu, les délais, les coûts, la qualité, les ressources humaines, les communications, les risques, les approvisionnements et les parties prenantes. Pour valider les acquis professionnels et pratiques de ses membres, le PMI ${ }^{\circledR}$ propose sept certifications professionnelles basées sur des aspects technico-pratiques du management de projet :

- Certified Associate in Project Management $(\mathrm{CAPM})^{\circledR}$;

- Project Management Professional (PMP) ${ }^{\circledR}$;

- Program Management Professional $(\text { PgMP })^{\circledR}$;

- Portfolio Management Professional $(\mathrm{PfMP})^{\mathrm{SM}}$;

- PMI Agile Certified Practitioner (PMI$\mathrm{ACP})^{\circledR}$;

- PMI Risk Management Professional (PMIRMP) $)^{\circledR}$;

- PMI Scheduling Professional (PMI-SP) ${ }^{\circledR}$.

\section{La démarche $\mathrm{ICB}^{\circledR} \mathrm{a}$ été la première à attirer l'attention de la communauté universitaire et professionnelle sur l'importance du management des parties prenantes dans les projets.}

Le deuxième corpus est porté par l'International Project Management Association (IPMA ${ }^{\circledR}$ ) à travers son corpus de compétence IPMA Competence Baseline $\left(\mathrm{ICB}^{\circledR}\right)$. IPMA ${ }^{\circledR}$ est un réseau international fédérant 57 associations professionnelles nationales de management de projet présentes sur les cinq continents et ayant son siège en Suisse. Au $27^{\mathrm{e}}$ congrès annuel de l'IPMA $^{\circledR}$ à Dubrovnik en Croatie en octobre 2013, IPMA ${ }^{\circledR}$ a réclamé 55000 membres individuels en plus de son réseau d'associations nationales ${ }^{11}$. Les auteurs reconnaissent que la démarche $\mathrm{ICB}^{\circledR}$ a été la première à attirer l'attention de la communauté universitaire et professionnelle sur l'importance du management des parties prenantes dans les projets. Selon la démarche $\mathrm{ICB}^{\circledR}$, le métier de chef de projet nécessite 46 compétences clés ${ }^{12}$ :

- 20 compétences techniques;

- 15 compétences comportementales et de leadership;

- 11 compétences contextuelles liées à l'environnement du projet.

IPMA $^{\circledR}$ certifie les professionnels qui font preuve de la maîtrise de ces compétences suivant quatre niveaux :

- IPMA® A : Certified Project Director;

- IPMA ${ }^{\circledR}$ B : Certified Senior Project Manager;

- IPMA ${ }^{\circledR}$ C : Certified Project Manager;

- IPMA ${ }^{\circledR}$ D : Certified Project Manage-ment Associate.

Le troisième corpus est développé par l'Association of Project Management $\left(\mathrm{APM}^{\circledR}\right)$ dont le siège est situé au Royaume-Uni. L'APM ${ }^{\circledR}$ valorise le corpus de connaissances APM ${ }^{\circledR}$ Body of Knowledge (APM $\left.\mathrm{BOK}^{\circledR}\right)$. En termes de participation, l'APM ${ }^{\circledR}$ revendique 21061 membres individuels et 500 organisations $^{13}$. L'APM BOK $^{\circledR}$ est une approche processus bâtie autour de sept principes, sept thèmes et sept processus. La particularité de cette démarche réside au niveau de son concept du « management par exception ». Cette disposition met en évidence le rôle clé du sponsor ou du promoteur dans le projet. Avec le management par exception, l'APM ${ }^{\circledR}$ considère que le management de projet est la fusion de trois verbes : diriger, gérer, livrer $^{14}$. Ainsi, grâce à l'APM ${ }^{\circledR}$, il a été possible d'établir la différence clé entre deux conceptions qui prêtes parfois à confusion : " management de projet » et « gestion des projets ». De ce fait, en plus du rôle de gestion, le manager de projet selon l'APMBOK $^{\circledR}$ endosse les rôles de direction et de 
livraison dans un contexte de projet ${ }^{15}$. L'APM ${ }^{\circledR}$ accrédite les professionnels qui maîtrisent son corpus par ses certifications PRoject IN Controlled environment (PRINCE2 ${ }^{\circledR}$ ) et ce, à deux niveaux :

- le niveau élémentaire ou PRINCE2® Foundation et

- le niveau élevé ou PRINCE2® Practitionner.
En plus de ces trois corpus dominants présentés cidessus, l'International Standard Organization (ISO) a créé récemment une norme internationale dont le but est de fédérer les différentes visions et de parvenir autant que faire se peut à un consensus global. Cette norme publiée en octobre 2012, est la « ligne directrice sur le management de projet ».

\section{ISO 21500, ALTERNATIVE NORMATIVE AUX CORPUS DOMINANTS DANS LA DISCIPLINE}

Le comité technique d'élaboration de la norme $I S O$ 21500 intitulée « lignes directrices sur le management de projet » a été mis sur pied en 2006 et a démarré ses travaux en février 2007 (ISO/PC 236). Comme le montre le Tableau 1 tiré du site web de Prescriptor, une firme africaine qui a participé aux travaux, ce comité était composé de 37 pays participants et de 15 pays observateurs. Présidé par la British Standards Institute, son secrétariat était tenu par l'American National Standards Institute (ANSI).

Dans son introduction, l'ISO 21500 mentionne : "La présente norme internationale fournit des orientations sur les concepts et les processus de gestion de projet qui sont importants pour la performance des projets » [Notre traduction] (p. 5) ${ }^{16}$.

Tableau 1 - Pays ayant contribués à l'élaboration de ISO 21500 (Source : Prescriptor-consulting.com)

\begin{tabular}{|c|c|}
\hline \multicolumn{2}{|c|}{ Participating Countries } \\
\hline Country & Starndard Body \\
\hline Argentina & IRAM - Instituto Argentino de Normalización y Certificación \\
\hline Australia & SA - Standards Australia \\
\hline Austria & ASI - Austrian Standards Institute \\
\hline Barbados & BNSI - Barbados National Standards Institution \\
\hline Belarus & BELST - State Committee for Standardization of the Republic of Belarus \\
\hline Brazil & ABNT - Associacão Brasileira de Normas Técnicas \\
\hline Cameroon & ANOR - Agence des Normes et de la Qualité \\
\hline Canada & SCC - Standards Council of Canada \\
\hline Chile & IINN - Instituto Nacional de Normalización \\
\hline China & SAC - Standardization Administration of China \\
\hline Costa Rica & INTECO - Instituto de Normas Técnicas de Costa Rica \\
\hline Denmark & DS - Fonden Dansk Standard \\
\hline Egypt & EOS - Egyptian Organization for Standardization and Quality \\
\hline Finland & SFS - Finnish Standards Association \\
\hline France & AFNOR - Association francaise de normalisation \\
\hline Germany & DIN - Deutsches Institut für Normung \\
\hline Greece & ELOT - Hellenic Organization for Standardization \\
\hline India & BIS - Bureau of Indian Standards \\
\hline Israel & SII - Standards Institution of Israel \\
\hline Italy & UNI - Ente Nazionale Italiano di Unificazione \\
\hline Japan & JISC - Japanese Industrial Standards Committee \\
\hline Korea, Republic of & KAT S - Korean Agency for Technology and Standards \\
\hline Luxembourg & $\begin{array}{l}\text { lLNAS - Institut luxembourgeois de la normalisation, de l'accréditation, de la sécurité } \\
\text { et qualité des produits et services }\end{array}$ \\
\hline Mexico & DGN - Dirección General de Normas \\
\hline Morocco & IMANOR - Institut Marocain de Normalisation \\
\hline Netherlands & NEN - Netherlands Standardization Institute \\
\hline Nigeria & SON - Standards Organisation of Nigeria \\
\hline Norway & SN - Standards Norway \\
\hline Portugal & IPQ - Instituto Português da Qualidade \\
\hline
\end{tabular}




\begin{tabular}{|c|c|}
\hline Romania & ASRO - Asociatia de Standardizare din România \\
\hline Russian Federation & GOSTR - Federal Agency on Technical Regulating and Metrology \\
\hline South Africa & SABS - South African Bureau of Standards \\
\hline Spain & AENOR - Asociación Española de Normalización y Certificación \\
\hline Sweden & SIS - Swedish Standards Institute \\
\hline Ukraine & DSSU - State Committee of Ukraine on Technical Requlation and Consumer Policy \\
\hline United Kingdom & B SI - British Standards Institution \\
\hline USA (Secretaria) & ANSI - American National Standards Institute \\
\hline \multicolumn{2}{|c|}{ Observing countries } \\
\hline Country & Starndard Body \\
\hline Belgium & NBN - Bureau de Normalisation \\
\hline Bulgaria & BDS - Bul garian Institute for Standardization \\
\hline Czech Republic & UNMZ - Czech Office for Standards, M etrologv and Testing \\
\hline El Salvador & OSN - Organismo Salvadoreño de Normalización \\
\hline Hong Kong, China & $\begin{array}{l}\text { ITCHKSAR - Innov ation and Technology Commission - Quality Services Division - } \\
\text { Product Standards Information_Bureaw }\end{array}$ \\
\hline Hungary & M SZT - Magyar Szabványügyi Testület \\
\hline Iceland & IST - Icelandic Standards \\
\hline Ireland & N SAl - N ational Standards A uthority of Ireland \\
\hline Lithuania & LST - Lithuanian Standards Board \\
\hline Malay sia & D SM - Standards M alay sia - Ministry of Science. Technology and Innov ation \\
\hline Pakistan & PSQCA - Pakistan Standards and Quality Control A uthority \\
\hline Poland & PKN - Polish Committee for Standar dization \\
\hline Saudi Arabia & SA SO - Saudi Standards. M etrology and Quality Organization \\
\hline Slovakia & SU TN - Slov ak Standards Institute \\
\hline Uruquay & UNIT - Instituto Uruguayo de Normas Técnicas \\
\hline
\end{tabular}

La nouvelle norme ISO 21500 a pour but de créer un cadre conceptuel et théorique commun et d'uniformiser les pratiques du management de projet dans la discipline face aux divergences relevées par les chercheurs en management de projet. ISO 21500 définit le management de projet comme étant " l'application des méthodes, des outils, des techniques et des compétences à un projet donné ». Les pays présentés au tableau 1 ont effectué plusieurs rencontres techniques dont les plus récentes se sont déroulées en France en février 2012 et en Afrique du Sud en juin 2012.

Le Tableau 2 compare le modèle ISO 21500 aux corpus dominants de connaissances en management de projet. Comme on peut le voir, les lignes directrices sur le management de projet (ISO 21500) présentent quelques similitudes avec les modèles existants, mais développent également des concepts nouveaux. Ainsi, pour se rapprocher de PMI $^{\circledR}$, l'ISO ${ }^{\circledR}$ considère les mêmes matières développées dans le $\mathrm{PMBOK}^{\circledR}$, tout en utilisant parfois des concepts différents. Par exemple, au lieu de parler des « corpus de connaissances » comme $\mathrm{PMI}^{\circledR}$, l'ISO $^{\circledR}$ préfère employer le concept de « groupes de sujets ». La norme ISO 21500 intègre la philosophie du « management par exception » développée dans le corpus de connaissances PRINCE $2^{\circledR}$. Cette démarche met l'accent sur le fait que le début de chaque étape devrait être précédé par la validation de la direction de l'entreprise. Ainsi, les décisions finales sur le contenu du produit incombent davantage à la direction plutôt qu'au chef de projet. Dans le cas où le chef de projet occuperait en même temps les rôles de directeur de l'entreprise réalisatrice et de gestionnaire du projet, la norme recommande fortement de procéder aux itérations de contrôle avant le lancement des nouvelles activités. ISO 21500 intègre enfin la philosophie des compétences de IPMA ${ }^{\circledR}$. Dans la définition de ses concepts, les lignes directrices insistent sur les trois compétences nécessaires aux chefs de projet : compétences techniques, comportementales et contextuelles. De plus, L'ISO 21500 intègre le management des parties prenantes comme nouveau groupe de sujet permettant d'établir une meilleure stratégie de management des acteurs dans les projets. Tout comme PMI $^{\circledR}$, IPMA ${ }^{\circledR}$ ou PRINCE2 ${ }^{\circledR}$, les 39 pages qui représentent le contenu de la norme ISO 21500 donnent une vue macroscopique des principales expressions nécessaires aux professionnels et chercheurs en management de projet ainsi que les environnements dans lesquels ils sont appliqués. Il s'agit d'uniformiser le langage et les expressions clés. Le management de projet selon ISO 21500 est basé sur deux piliers principaux : les concepts et les processus. ISO 21500 Enfin, ISO 21500 encourage le transfert d'information entre les membres de l'équipe d'un projet. 
Tableau 2 - Compilation de quelques différences et similitudes entre ISO 21500, PMBOK ${ }^{\circledR}$, ICB $^{\circledR}$ et PRINCE2 ${ }^{\circledR}$

\begin{tabular}{|c|c|c|c|c|}
\hline & ISO $21500^{\circledR}$ & PMBOK $^{\circledR} 5$ & PRINCE $2^{\circledR}$ & IPMA CB ${ }^{\circledR}$ \\
\hline $\begin{array}{l}\text { Groupes de } \\
\text { processus }\end{array}$ & 5 groupes de processus & 5 groupes de processus & $\begin{array}{l}4 \text { groupes de } \\
\text { processus }\end{array}$ & $\begin{array}{l}3 \text { groupes de } \\
\text { compétence }\end{array}$ \\
\hline Sujets & 10 groupes de sujets & $\begin{array}{l}10 \text { domaines de } \\
\text { connaissance }\end{array}$ & 7 thèmes & $\begin{array}{l}10 \text { domaines de } \\
\text { connaissance }\end{array}$ \\
\hline Processus & 39 processus & 47 processus & 7 processus & 46 processus \\
\hline $\begin{array}{l}\text { Groupes de } \\
\text { processus }\end{array}$ & $\begin{array}{l}\text { 1. Lancement } \\
\text { 2. Planification } \\
\text { 3. Mise en œuvre } \\
\text { 4. Maîtrise } \\
\text { 5. Clôture }\end{array}$ & $\begin{array}{l}\text { 1. Démarrage } \\
\text { 2. Planification } \\
\text { 3. Exécution } \\
\text { 4. Surveillance et maîtrise } \\
\text { 5. Clôture }\end{array}$ & $\begin{array}{l}\text { 1. Élaborer un projet } \\
\text { 2. Diriger un projet } \\
\text { 3. Initialiser un projet } \\
\text { 4. Contrôler une } \\
\text { séquence } \\
\text { 5. Gérer la livraison } \\
\text { du produit } \\
\text { 6. Gérer une limite de } \\
\text { séquence } \\
\text { 7. Clore le projet }\end{array}$ & $\begin{array}{l}\text { 1. Compétences } \\
\text { techniques } \\
\text { 2. Compétences } \\
\text { comportementales } \\
\text { 3. Compétences } \\
\text { contextuelles }\end{array}$ \\
\hline Thématiques & $\begin{array}{l}\text { 1. Intégration } \\
\text { 2. Parties prenantes } \\
\text { 3. Contenu } \\
\text { 4. Ressources } \\
\text { 5. Délais } \\
\text { 6. Coûts } \\
\text { 7. Risques } \\
\text { 8. Qualité } \\
\text { 9. Approvisionnement } \\
\text { 10. Communication }\end{array}$ & $\begin{array}{l}\text { 1. Intégration } \\
\text { 2. Parties prenantes } \\
\text { 3. Contenu } \\
\text { 4. Ressources humaines } \\
\text { 5. Délais } \\
\text { 6. Coûts } \\
\text { 7. Risques } \\
\text { 8. Qualité } \\
\text { 9. Approvisionnement } \\
\text { 10. Communication }\end{array}$ & $\begin{array}{l}\text { 1. Cas d'affaire } \\
\text { 2. Organisation } \\
\text { 3. Qualité } \\
\text { 4. Plan } \\
\text { 5. Risques } \\
\text { 6. Changement } \\
\text { 7. Progression }\end{array}$ & $\begin{array}{l}\text { 1. Organisation } \\
\text { 2. Risques et } \\
\text { opportunités } \\
\text { 3. Qualité } \\
\text { 4. Parties prenantes } \\
\text { 5. Contenu et livrables } \\
\text { 6. Délais et phases } \\
\text { 7. Ressources } \\
\text { 8. Coût et finances } \\
\text { 9. Approvisionnement } \\
\text { et contrats } \\
\text { 10. Modification } \\
\text { 11. Information et } \\
\text { documentation } \\
\text { 12. Communication } \\
\text { 13. Leadership } \\
\text { 14. Éthique } \\
\text { 15. Santé, sécurité et } \\
\text { environnement } \\
\text { 16. Finance et Droit }\end{array}$ \\
\hline
\end{tabular}

\section{CONCLUSION}

Le management de projet n'est pas nouveau. Comme nous venons de le démontrer, dans la pratique, le management de projet s'exerce depuis des siècles. Toutefois, il est possible de concevoir que les bases théoriques et idéologiques du management de projet datent depuis la venue des corpus de connaissances, au courant des années 1960.

Dans cette lancée, plusieurs corpus ont fait leur apparition. De ceux-ci, trois dominent le terrain pratique et théorique de la discipline : le $\mathrm{PMBOK}^{\circledR}$ de PMI $^{\circledR}$, l'ICB $^{\circledR}$ de IPMA $^{\circledR}$ et l'APMBOK $^{\circledR}$ de l'APM $^{\circledR}$. En outre, si ces corpus sont perçus par la plupart des professionnels et des chercheurs comme les principaux dans la discipline du management de projet, l'on est quand même loin d'ignorer la montée en puissance de certains corpus de connaissances propriétaires issus des associations nationales de normalisation en management de projet. 
Maintenant, la venue de la norme internationale ISO 21500 qui trace les lignes directrices en management de projet contribue à encourager l'utilisation des mêmes concepts et processus quel que soit l'environnement géographique, le niveau d'expérience ou le type de projet. Toutefois, si nous considérons la norme ISO 21500 comme une alternative normative aux corpus dominants en management de projet, une question demeure : la norme ISO 21500 suffira-t-elle à normaliser les conceptions et les positionnements épistémologiques? En regardant de près, les lignes directrices sur le management de projet ne proposent aucun outil, encore moins aucune technique aux praticiens. Ce qui peut constituer d'entrée de jeu une ouverture capable de favoriser la perpétuité des corpus existants

\section{BIBLIOGRAPHIE}

${ }^{1}$ Fayol, H. (1916). « Administration industrielle et générale », Bulletin de la Société de l'Industrie Minérale, $\mathrm{n}^{\mathrm{o}} 10$, p. 5-164, Rééditions régulières par Dunod depuis 1918.

${ }^{2}$ Morris, P. W. G. et Hough (1987). The Anatomy of Major Projects: A Study of the Reality of Project Management, Wiley.

${ }^{3}$ Snyder, J. R. et Kline, S. (1987). « Modern Project Management: How Did We Get Here - Where Do We Go?».

${ }^{4}$ Kwak, Y. H. (2003). " The Story of Managing Projects ».

${ }^{5}$ Gerard Blokdijk (2007). Project Management 100 Success Secrets, Lulu.com.

${ }^{6}$ Goldratt, E. M. (1997). Critical Chain. North River Press, Great Barrington, MA.

${ }^{7}$ Le Moigne J. L. (1990). " Épistémologies constructivistes et sciences de l'organisation c, dans Martinet A.C. (Editeur), Epistémologies et sciences de gestion, Economica.

${ }^{8}$ Morris, P. W. G. et Smyth, H. J. (2007). « An epistemological evaluation of research into projects and their management : Methodological issues », IJPM, p. $423-436$.

${ }^{9}$ PMI (2013). « PMI total numbers ». PMIToday, 30 septembre 2013, p. 5.

${ }^{10}$ Project Management Institute (2013). A guide to the project management body of knowledge (PMBOK guide), Newton Square, Penns., Project Management Institute.

${ }^{11}$ IPMA (2013). $27^{e}$ congrès annuel de l'IPMA ${ }^{\circledR}$ à Dubrovnik en Croatie en octobre 2013.

${ }^{12}$ International Project Management Association (2006).

IPMA Competence Baseline, version 3.0.
${ }^{13}$ APM (2013). APM Annual Report and Accounts 2012/13.

${ }^{14}$ APM (2010). A History of the Association for Project Management.

${ }^{15}$ Office of Government Commerce (2008). Managing Succesful Projects with PRINCE2.

${ }^{16}$ International Standard Organization (2012). ISO : 21500, Lignes directrices sur le management de projet. 